\title{
Encuentros entre la Pedagogía crítica y la Teoría de la resistencia
}

\author{
Encounters: between critical pedagogy and the theory of resistance
}

Gustavo Villamizar Acevedo ${ }^{a}$ ORCID: 0000-0002-8886-1993

Recibido: 6/12/2019• Aprobado: 26/01/2020

Cómo citar: Villamizar Acevedo, G. (2020). Encuentros entre la Pedagogía crítica y la Teoría de la resistencia. Cienciay Educación, 4(1), 83-90. Doi: https://doi.org/10.22206/cyed.2020.v4i1.pp83-90

\begin{abstract}
Resumen
En un momento de la historia en el que la educación está al servicio del neoliberalismo es necesario replantear su función. Para tal fin se analizan los fundamentos de la Pedagogía crítica y la Teoría de la resistencia y sus puntos de convergencia. Se realiza un recuento por los planteamientos de Habermas, en la Teoría de la acción comunicativa, seguido por los establecidos por Henry Giroux, quien invita a los maestros a analizar el papel de los medios de comunicación como soportes de la ideología dominante y oponerse a ellos a través de una Pedagogía liberadora. Asimismo, el artículo aborda la Teoría de la resistencia, línea de pensamiento que se encuentra con la teoría de Paulo Freire, la cual ve a los docentes como promotores de una educación liberadora. Bajo esta consideración, la Pedagogía crítica es vista como una pedagogía que busca la formación del pensamiento crítico y que considera el aula como un escenario de resistencia.
\end{abstract}

Palabras clave: Pedagogía crítica; Teoría crítica; resistencia; medios de comunicación.

\begin{abstract}
At this point in history, when education is at the service of neoliberalism, it becomes necessary to rethink its function. To do so, this paper analyzes the foundations of critical Pedagogy, the theory of resistance, and points where they convergence. It starts with an overview of Habermas's communicative action theory. It continues presenting Henry Giroux's analysis of the role of media in supporting the dominant ideology and his open invitation, to teachers, to oppose this role through liberating pedagogy. The article also deals with the theory of resistance, an approach that meets Paulo Freire's view of teachers as promoters of a liberating education. From this approach, critical pedagogy is seen as a pedagogy that seeks the formation of critical thinking and considers the classroom as the scenario of resistance.
\end{abstract}

Keywords: Critical pedagogy; Critical theory; resistance; mass media.

a Universidad Pontificia Bolivariana Bucaramanga, Colombia. Correo-e: gustavo.villamizar@upb.edu.co 


\section{Introducción}

De acuerdo con Giroux (2019), la educación ha sido puesta al servicio del neoliberalismo, que la ha convertido en una proveedora de mano de obra. Esta afirmación sirvió de base para la elaboración de este artículo reflexivo que tiene por objetivo analizar los presupuestos teóricos de la Pedagogía crítica y de la Teoría de la resistencia, así como los puntos en las cuales convergen, bajo la consideración que ambos enfoques buscan formar personas comprometidas con la transformación social a partir del desarrollo del pensamiento crítico. Este artículo consta de tres apartados; el primero aborda los principios de la Pedagogía crítica, donde se plasman los fundamentos filosóficos y epistemológicos que sustentan dicha teoría. En el segundo se analiza el rol de los medios de comunicación y su relación con las instancias de poder; y en el tercero se hace un acercamiento a la Teoría de la resistencia, sus contribuciones para la emancipación social y sus encuentros con la Pedagogía de la liberación propuesta por Paulo Freire.

\section{Principios de la Pedagogía crítica}

La Pedagogía crítica se desarrolló alrededor de 1970 y se visualiza, de acuerdo con Carr \& Kemmis (1988), como un proceso de cambio social que se debe realizar de forma colectiva, es decir, con todos los participantes en la acción educativa: docentes, estudiantes, padres de familia y comunidad en general.

Este enfoque pedagógico aplica los principios teóricos de la Escuela de Frankfurt, específicamente los de Habermas (2004), estructurados en su reconocida obra Teoría de la Acción Comunicativa, donde postula que las personas usan el conocimiento para ponerse de acuerdo.

$\mathrm{Al}$ enmarcarse en la acción comunicativa, es indispensable considerar los presupuestos de Habermas relacionados con la investigación social, sobre la cual este filósofo alemán (como se citó en Vasco, 1985) planteó la existencia de tres formas de efectuarlas, que se diferencian entre sí por el interés que las mueve y que corresponderían a:
1. El interés técnico, el cual busca el control y la regulación de los objetos, respondiendo a un modelo de ciencia fundamentado en lo empírico analítico y que en el plano metodológico es de tipo hipotético deductivo.

2. El interés práctico, que tiene la intención de guiar la comprensión humana a través de los principios de las ciencias hermenéuticas, por eso sus productos son de carácter interpretativo y "anima a los educadores a hacer interpretaciones de las circunstancias sociales y educativas actuales en situaciones reales concretas y a tomar decisiones sobre la base de su razonamiento práctico" (Kemmis, 1998, p. 87).

3. El interés emancipador, que tiene como intención liberar a los seres humanos "de las ideas falsas, de las formas de comunicación distorsionada y de las formas coercitivas de la relación social que constriñen la acción social humana" (Kemmis, 1998, p. 87).

Teniendo en cuenta lo consignado, se nota la importancia que este enfoque pedagógico otorga a la comunicación, al análisis hermenéutico y a la liberación. El primero de estos principios, el de comunicación, corresponde a la producción de Habermas y los de análisis hermenéutico y liberación a la Escuela de Frankfurt.

Para la comunicación, el instrumento central de trabajo es la acción comunicativa; según sus supuestos, utilizarla favorece la creación de ciertas situaciones que posibilitan las superaciones de las relaciones asimétricas, resultantes del establecimiento de niveles entre los que participan en la escuela: maestros, estudiantes y comunidad.

Con relación al análisis hermenéutico, es indispensable verlo como uno de sus principales criterios metodológicos, el cual según Nadal (2019, p. 194) desde la perspectiva heideggeriana se concibe "como una filosofía universal de la interpretación”. El objetivo del análisis hermenéutico es "llegar al sentido", el cual se "construye a partir de una primera proyección de los significados propios del sujeto que estudia, para después en sucesivas interpretaciones ir descubriendo otras dimensiones y siempre en relación con el entorno" (Sáenz, 2008, p. 13). 
Respecto al papel liberador o emancipatorio, que toma Habermas directamente de la Teoría crítica, fue propuesto por diversos teóricos de este enfoque y tiene como objetivo la creación de un pensamiento crítico, que debe tener un carácter develador, que descubra "lo no-dicho", es decir, aquellos aspectos de la realidad no establecidos en los discursos oficiales, pero que hacen parte de la realidad, no buscando propiciar con esto discusiones interminables en torno a las disposiciones "legales", sino permitirle al hombre alcanzar su libertad. Por tal razón, el aula es considerada un lugar de resistencia en firme oposición al pensamiento dominante (Santamaría, Benítez, Sotomayor \& Barragán, 2019).

Para alcanzar el pensamiento crítico, este enfoque recalca el papel que tiene la ciencia en la sociedad, el cual de ninguna manera es neutral, ya que el conocimiento validado responde a intereses sociohistóricos $y$, por tanto, reconoce la jerarquía de valores establecidos, pues las ideologías permean las teorías y por eso una de las tareas de la Teoría crítica es analizar y criticar la ideología dominante (Kemmis, 1998).

La Teoría crítica pretende ir más allá de la interpretación del mundo, por eso brinda a los estudiantes herramientas que les permitan transformarlo, entre las cuales se destacan la mayéutica socrática y la investigación-acción. La primera de estas herramientas se encuentra relacionada con la "acción comunicativa", que en este marco se denomina "acción educativa", y se relaciona con "una situación ideal de habla", según Habermas (como se citó en Sáenz, 2008); esta situación se presenta cuando los participantes, como agentes activos, no solo pueden participar en los actos comunicativos sino que están en condiciones para afirmar o refutar los argumentos asumidos como válidos.

Con respecto a la investigación acción, este enfoque parte de la contextualización de un problema y pretende responder cuestionamientos como: ¿qué está sucediendo?, ¿en qué sentido es problemático”, ¿qué puedo hacer yo al respecto?, preguntas que buscan dinamizar la situación activando el ciclo de planificación-acción-evaluación.

\subsection{Medios de comunicación y Teoría de la resis- tencia}

Por otra parte, es indispensable analizar el papel de los medios de comunicación en el ejercicio de informar, a partir del interés por parte de los centros de poder. Actualmente, se plantea en algunos países la disyuntiva de quién controla los contenidos en los distintos medios, ya que como afirma Giroux (1997, p. 67) la historia muestra que "bajo el régimen del positivismo, la razón siente una admiración reverencial por los hechos (...) Bajo el régimen del positivismo, la razón se detiene inevitablemente antes de la crítica"; ante tal premisa: ¿qué es lo objetivo en la información mediada?, o ante los simples hechos ¡se puede formar a los jóvenes con postura crítica? Son cuestiones que se plantean respecto al papel de los medios de comunicación en la labor educativa.

Los medios de comunicación en la era del positivismo consideran que lo "objetivo" es la columna vertical del conocimiento científico y que al aplicarse en el hecho social o cotidiano, como lo percibe Giroux (1997, p. 69), "se separan de los valores, la objetividad socava la crítica, y la idea de que la esencia y la apariencia pueden no coincidir carece de sentido".

Esta visión influyó en la formación de una conciencia colectiva donde la "razón" prevalece sobre la crítica, pero ¿los medios contribuyen a la formación de una conciencia social que sirve a una clase dominante de la sociedad? El solo hecho de que los medios de comunicación se presenten como entes objetivos ante una confiada audiencia no le asigna el papel de formadores de personas de pensamiento crítico; aunque el docente no debe permanecer neutro ante los mensajes que invade la mentalidad de los jóvenes, ante esta perspectiva si el docente toma una actitud pasiva, los medios asumen el papel de formadores.

En tal caso, los medios como simples presentadores de los hechos no contribuyen, según lo expresado por Giroux ((1997, p. 76), a presentar "una concepción dialéctica de la sociedad y la teoría sostendría que la observación no puede tomar el lugar de la reflexión y la comprensión críticas"; es decir, el medio objetivo no existe, pues debe ir más allá de presentar los hechos; su papel al igual que el del docente es preparar a las 
personas para que tengan criterios de base para establecer la capacidad de diálogo y de participación. Solo de esta forma podrán acercar a las personas a su emancipación.

Dentro de la programación de los medios, como la televisión, su "valor reside en la aptitud para establecer las posibilidades del pensamiento y la práctica reflexivos por parte de quienes la usan; en el caso de los docentes, se convierte en un instrumento invalorable de crítica y comprensión”; por lo tanto, los medios en la actualidad deben utilizar las ventajas de la tecnología en los procesos de diálogo para estimular el pensamiento libre.

Es fundamental la participación de los medios de comunicación en el desarrollo cultural de la sociedad, pues como bien lo formula Giroux (1997, p. 80) el poder de las clases dirigentes se establecía "mediante el imperio del consenso, y se transmitía por conducto de instituciones culturales como las escuelas, la familia, los medios masivos de comunicación, las iglesias”. Por tal razón, los medios de comunicación determinan las creencias o los patrones de conducta dirigidas a la transformación social, o a la destrucción de lo establecido, de ahí su especial atención por los gobiernos de turno, los líderes sociales, económicos y culturales.

Los medios de comunicación, como la televisión, al ofrecer únicamente entretenimiento solo alcanza un objetivo: poner la audiencia al servicio de los intereses económicos o políticos, sin libertad de conciencia para tomar sus decisiones. Situación que se incrementa al invadir la privacidad del hogar, a través de la radio, la prensa, la televisión y el internet, "la colonización del lugar de trabajo se complementaba ahora con la colonización de todas las otras esferas culturales" (Giroux, 1997).

Por otra parte, los educandos no visualizan cuál es la ideología predominante en su contexto; según Giroux (1997, p.124), "los alumnos no pueden conocer la ideología por el mero hecho de que se les enseñe que los significados se construyen socialmente en los medios masivos de comunicación y otros aspectos de la vida diaria"; no establecen criterios de juicio de conciencia crítica, porque los medios, la familia y su entorno más inmediato conforma su cosmovisión limitada.
Ante esta situación, Giroux (1983) invita a un proceso de resistencia contra la ideología y la política cultural de los grupos dominantes, expresados en la escuela a través del "currículum oculto"; por tanto, la Pedagogía crítica estudia cómo clase y cultura se integran para ofrecer descripciones según políticas culturales vistas desde la semiótica y los sistemas de significado que conforman el campo cultural del oprimido, buscando analizar los elementos contrahegemónicos existentes en ese campo y la forma como son incorporados dentro de la cultura dominante, despojándolos de su fuerza política, es decir convirtiéndolos en discursos políticamente inofensivos.

\subsection{Acerca de la Teoría de la resistencia}

La resistencia desde la perspectiva pedagógica planteada por Giroux ofrece importantes contribuciones para indagar acerca de la relación escuela-sociedad. Esta teoría está llamada a erigir una pedagogía radical de emancipación social desde el proceso de la escolarización, donde la reproducción no es solo vista como el reflejo de la sociedad dominante o de fuerzas externas (familia, escuela) imposibles de retar o modificar, sino como resultado de fuerzas internas que inconscientemente promueven la resistencia y que soportan un constante ajuste con elementos de oposición parcialmente percibidos (divisiones sociales y sexuales, por ejemplo).

Aunque Giroux (1998) centra su análisis en el sistema de educación estadounidense, se fundamenta en las teorías de reproducción sociocultural de autores como Althusser, Bowles y Gintis, Bourdieu, Passeron y Bernstein, quienes plantearon los cimientos para la creación de una teoría que permita explicar cómo se presenta la reproducción social y cultural en la escuela, así como la generación de diversos tipos de resistencia por parte de los grupos subordinados de la sociedad escolar y el papel que en ellos juega la escuela, así como lo que los educadores deben saber y hacer ante estos hechos.

La teoría de la reproducción social devela la función de la escuela como sostén de las relaciones capitalistas de producción a través de la formación de habilidades laborales y la producción de conciencias compatibles 
(actitudes, valores y normas) con los intereses de la sociedad dominante; así los estudiantes de diversas clases sociales aprenden lo que requieren para ocupar los lugares que les corresponden según su clase en la división ocupacional del trabajo, yendo en desmedro del desarrollo de habilidades que permitan ir más allá del límite establecido (Giroux, 1998).

Althusser, Bowles y Gintis analizan los diferentes aspectos del proceso de reproducción dados en el vínculo economía-escuela, relación que permite el mantenimiento y la reproducción de los países industrializados de Occidente, así como la forma en que los sistemas sociopolíticos ubican a los seres humanos de acuerdo a la fuerza de trabajo y a la dinámica de la interacción de clase bajo el sistema capitalista. Las relaciones sociales en el salón de clase sirven para inculcar tales actitudes y disposiciones a los estudiantes, tal como señalan Bowles \& Gintis (Giroux, 1998).

Sin embargo, como lo afirma Giroux (1998), estos autores no tienen en cuenta que el conocimiento en las aulas está influido tanto por la cultura de la escuela y de la clase como por el significado que los maestros y los estudiantes le otorgan durante el estudio, además no explican cómo el conocimiento es producido, ni el rol de la conciencia, la ideología, las contradicciones y las formas de resistencia durante la escolarización.

Bourdieu y Passeron desarrollaron una sociología del currículum que vincula cultura, clase y dominación en sus teorías de reproducción cultural (Giroux, 1998). Las escuelas, según ellos, no son solo un espejo que reproduce la sociedad, sino que son relativamente autónomas (pretexto de independencia y neutralidad) y solo están indirectamente influenciadas por las instituciones políticas y económicas poderosas.

La escuela es vista como un universo amplio de instituciones simbólicas (violencia simbólica) donde las relaciones existentes de poder se reproducen sutilmente a través de una cultura dominante. La cultura pasa a ser el vínculo mediador entre los intereses de la clase dirigente y la vida cotidiana para establecer una definición del mundo social de acuerdo con sus intereses. Para analizar lo que sucede en la escuela crean conceptos como: capital cultural (herencia lingüística y cultural familiar), que permite identificar los determinantes generadores tanto de ventajas como de desventajas que tendrán socialmente y habitus como esquemas de pensamiento y cuerpo internalizados que estructuran experiencias sociales.

Una de las críticas de Giroux (1998) al legado de Bourdieu se cimienta en que la producción cultural de la clase trabajadora, al no ser homogénea, es un simple reflejo de la cultura dominante y no se reconoce su vínculo con la reproducción por medio del proceso de incorporación o acomodamiento. También, le critica el hecho de darle un tratamiento unilateral a la ideología, en el sentido de que las ideologías dominantes no son solo transmitidas por las escuelas en un acto pasivo, sino que además son enfrentadas y reconstruidas por los miembros de la comunidad escolar.

Bernstein, según Giroux (1998) en su análisis sobre el rol de la educación en la reproducción cultural de las relaciones de clase, se refiere a un código educacional o sistema de mensajes que organiza la forma en que las diferentes manifestaciones de la experiencia escolar (currículum, pedagogía, evaluación) median con la autoridad y el poder. Pero, Giroux confiere a estos códigos el carácter de limitados, al minimizar tanto la resistencia y la lucha contrahegemónica como el papel de los actores sociales como agentes de cambio.

Giroux, desde un análisis de la teoría educativa, determinó que esta desarrolló un análisis teórico estructural e interaccional del proceso de escolarización y suministró nuevos modos de descubrir las ideologías e intereses que caracterizan la cotidianidad en las escuelas, a través de los modos de análisis de un currículum oculto, que como lo indica el mismo nombre no se evidencia en la teoría y la práctica educativa, al hacerse evidente una separación de poder y conocimiento, disociando la cultura de la política.

El trabajo de Giroux se mueve entre una sólida perspectiva teórica crítica y reflexiva de la escolarización y una práctica cuya especificidad reside, a su vez, en la teoría como centro de gravedad. La teoría toma su forma de la práctica y su costo le otorga "la habilidad de ofrecer la reflexión necesaria para interpretar a la experiencia concreta que es el objeto de investigación” (Giroux, 1998, p. 133).

Giroux, refuerza este hecho con el proceso de investigación. Para él la teoría debe estar interconectada con 
la experiencia, representando distintos momentos analíticos, y no deberían presentarse enfrentamiento entre ellas, de acuerdo a lo planteado por Horkheimer (como se citó en Giroux, 1998).

Esta implicación entre la teoría como mediadora en los estudios de la resistencia neomarxista y su especificad en la práctica representa para Giroux el punto de partida de su análisis de una teoría pedagógica crítica (radical) de reproducción y de la resistencia, aludiendo las inconsistencias de los postulados teóricos de los autores que estudia, por carecer de profundidad en sus bases teóricas.

Giroux entra en oposición con los autores que estudió, ya que estos no hacen uso de ejemplos válidos de hechos de dominación coherentes u homogéneos; por ejemplo, en cuanto a la omisión de problemas de género y raza (se reduce solo a hombres, prácticas sexistas, relego de necesidades y deseos por parte de los educadores), a reducción de categorías y nociones descriptivas que caracterizan la teoría de la resistencia, el análisis del discurso y las prácticas en el salón de clase, referido a la forma de producción de subjetividades (no visibles, subversivas, menos obvias, progresistas, actitudinales) o situaciones inmediatas suscitadas en un salón de clase, sin considerar la resistencia como un hecho en construcción con principios de determinación individual y social que puede terminar siendo acomodación, conformismo o actos de autoindulgencia, más que de resistencia u oposición a ideologías de dominación que se erigen desde una herencia histórico-social-familiar y de conciencia e ideología represivas personales.

Para Giroux, la resistencia debe promover el pensamiento crítico y la acción reflexiva, así como estimular la lucha política colectiva ante el poder y el determinismo social, yendo más allá de lo inmediato, buscando develar lo que hay tras las escenas: su lógica oculta.

La resistencia desde esta perspectiva educativa analiza la cultura de la escuela dentro de un escenario en constante lucha y enfrentamiento, donde el conocimiento educativo, los valores y las relaciones sociales están ubicados en contextos antagónicos. Los elementos de la resistencia les permiten a los estudiantes vivir una gran diversidad de experiencias por medio de las cuales puedan manifestarse y valorar las dimensiones positivas de su cultura e historia.

Estos supuestos se sintonizan y concuerdan con los propósitos de Freire (2005) en su Pedagogía del oprimido, al plantear cómo la práctica pedagógica debe ser elaborada con el sujeto dominado y no para él, reflexionando sobre la opresión y sus causas, de la cual emergerá el compromiso para luchar por su liberación, motivo central de esta pedagogía.

En la Teoría de la resistencia emergen las categorías centrales de la problemática de la resistencia postuladas por Giroux (1998, p. 144): "la intencionalidad, la conciencia, el significado del sentido común y la naturaleza y valor del comportamiento no discursivo", las cuales asumen la emancipación como concepto guía, con la intención clara de ser "una crítica de la dominación y ofrezca las oportunidades teóricas para la auto reflexión y lucha en el interés de la emancipación propia y de la emancipación social“" (Giroux, 1998, p. 145), en la que esta última armonice con la "cualidad relacional del ser humano" que señala Freire como aspecto esencial de la existencia humana.

En la misma línea del pensamiento de Giroux, aunque analizando la situación educativa desde otro contexto, Freire (2005) apoya sus postulados en el avance hacia una pedagogía crítica o radical denominada por él Pedagogía del oprimido o Pedagogía liberadora, la cual reacciona ante un sistema de dominación capitalista, que incorpora a los individuos a través de la escolarización.

Freire estructura sus fundamentos en diversos enfoques del siglo $\mathrm{xx}$ aplicados al quehacer educativo (psicológico, sociológico). A su vez, va más allá de la amplitud social y cultural del individuo, sin ser individualista, analizando la estructura de pensamiento (condicionadas) y las contradicciones (adherencia al opresor) padecidas por el "oprimido", desde las relaciones sociales y la existencia humana (cualidad relacional), para lo cual se apoya en la interdisciplinariedad, en estudios de corte marxista y psicoanalíticos y en situaciones experienciales, con el fin de intentar comprender y explicar las formas, el sentimiento y hasta los miedos generados por la opresión, consciente o inconsciente, adquiridas, manifestadas y hasta encubiertas por las sociedades. 
La opresión, para Freire, desde la perspectiva psicológica, constituye uno de los más profundos sufrimientos que pueda vivir un ser humano, en lo material, económico, político, espiritual, educativo, ya que genera en él un agravante de contradicción y problema existencial. En esta visión del mundo y del individuo, Freire (2005) conduce su pedagogía del opresor a una pedagogía que pueda "liberarlo" del carácter dual de opresor y oprimido, de la acomodación y adaptación que él mismo alberga al adherir a las formas del ser y actuar del opresor. Mientras esta dualidad permanezca, en la cual "ser es parecer y parecer es parecerse con el opresor", es decir, la identificación con su contrario, es imposible llegar a una pedagogía con una visión liberadora o emancipadora.

Desde esta perspectiva, Freire (1971) apunta a ver el proceso educativo como el iniciador y promotor de la lucha por la liberación como conquista, mas no como donación ni generosidad del opresor, sino desde el currículum escolar y el entendimiento de una relación positiva, asertiva y conciliadora entre educandos y educadores, de carácter recíproco, es decir de "todos con todos".

La explicación de Freire alude a una educación netamente humanizante, opuesta a una educación rígida dominada por la autoridad y los "saberes" del educador, unilateral y direccionada (educación bancaria), que se expresa como una suerte de prohibición a ser feliz y, por lo tanto, opresiva, en decadencia a las necesidades específicamente humanas (Sánchez, Sandoval, Goyeneche, Gallego \& Aristizabal, 2017; Mora, 2018; Niño, 2019).

Según Freire, una tarea fundamental del educador debe ser la identificación y la superación de la rigidez psicológica que lo lleva a oponerse al diálogo. En lugar de eso, es necesario incentivar la horizontalización de las relaciones humanas, la cual, sin llegar a ser utópica, apunta a una doble visión y transformación del hecho educativo, tanto en el interior de la persona como en las estructuras sociales. "La concepción de una persona esencialmente libre, comprometida con unos valores y abierta al otro caracteriza la visión de Freire, donde la transformación de lo humano resulta el rasgo vital y definitorio de la existencia" (Santos, 2008, p. 172).

\section{Conclusiones}

La Pedagogía crítica surge y se desarrolla durante el siglo xx. Su estructura se da a partir de los principios de la Escuela de Frankfurt, fundamentalmente a partir de la obra de Habermas Teoría de la Acción Comunicativa. Este enfoque pedagógico considera indispensable analizar el papel de los medios de comunicación, los cuales responden a las instancias de poder; ante esta situación promueven la resistencia por medio del desarrollo del pensamiento crítico y la acción reflexiva, propósitos en los cuales se encuentran con los planteamientos de Paulo Freire, quien postuló una pedagogía liberadora, que concibe la educación como un proceso de lucha por la liberación.

Tanto Giroux como Freire instan a los educadores a ser precisos sobre lo que la resistencia es, para que así estructuren una pedagogía crítica o "liberadora”, fundamentada en razonamientos teóricos rigurosos y útiles, enmarcada como un modo de discurso de análisis de la conducta de oposición que requiere de un conjunto de supuestos acerca de la escolarización, negados tanto por la teoría tradicional de la escuela como por la de la reproducción social y cultural.

\section{Referencias}

Carr, W. \& Kemmis, S. (1988). Teoría crítica de la enseñanza. La investigación-acción en la formación del profesorado. Barcelona: Martínez Roca.

Freire, P. (2005). Pedagogía del oprimido. México D.F.: Siglo XxI.

Freire, P. (1971). La Educación como práctica de la libertad. México D. F.: Siglo XxI.

Giroux, H (1983). Teorias de la reproducción y la resistencia en la nueva sociología de la educación: un análisis crítico. http://files.doctorado-en-educacion-2-cohorte.webnode.es/200000079-7133380f5/teorias $\% 20 \mathrm{de} \% 20 \mathrm{la} \% 20$ reproduccion $\% 20 \mathrm{y} \% 20$ la\%20resistencia_Giroux.pdf

Giroux, H (1997). Pedagogía y Politica de la esperanza. Buenos Aires: Amorrortu Editores.

Giroux, H. (1998). Teoría y Resistencia en Educación. México D.F.: Siglo XxI.

Giroux, H. (2019). Hacia una pedagogía de la esperanza educada bajo el capitalismo de casino. Pedagogía y Saberes, 50, 153-158. 
Habermas, J. (2004). Teoría de la Acción Comunicativa. Madrid: Trotta.

Kemmis, S. (1998). El currículum: más allá de la teoría de la reproducción. Madrid: Morata.

Mora, R. (2018). La pedagogía crítica y la educación actual. Revista Ciencias y Humanidades, 7(7), 175-190.

Nadal, J. (2019). Observaciones sobre encuentros entre hermenéutica, pragmática y análisis del discurso. Revista Mexicana de Ciencias Politicas y Sociales, 236, 193-216.

Niño, Y. (2019). Problematizar lo humano en educación. La dimensión política y el concepto de pensamiento crítico en la pedagogía de Freire y Giroux. Pedagogia y Saberes, 51, 133-144.

Sáenz, A. (2008). Teoría Crítica y Educación. https:// crisiseducativa.files.wordpress.com/2008/03/ teoria-critica-y-educacion.pdf
Sánchez, N., Sandoval, E., Goyeneche, R., Gallego, D. \& Aristizabal, Y. (2017). La pedagogía crítica desde la perspectiva de Freire, Giroux, y Mclaren: su pertinencia en el contexto de Colombia y América Latina. Revista Espacios, 39(10), 41-51.

Santamaría, J., Benítez, C., Sotomayor, S. \& Barragán, L. (2019). Pedagogías críticas: criterios para una formación de docentes en investigación pedagógica. Educação \& Sociedade, 40, 1-20.

Santos, G (2008). Ideas filosóficas que fundamentan la pedagogía de Paulo Freire. Revista Iberoamericana de Educación, 46, 155-173.

Vasco, C. (1985). Tres Estilos de Trabajo en las Ciencias Sociales. Santafé de Bogotá: Universidad Nacional de Colombia. 